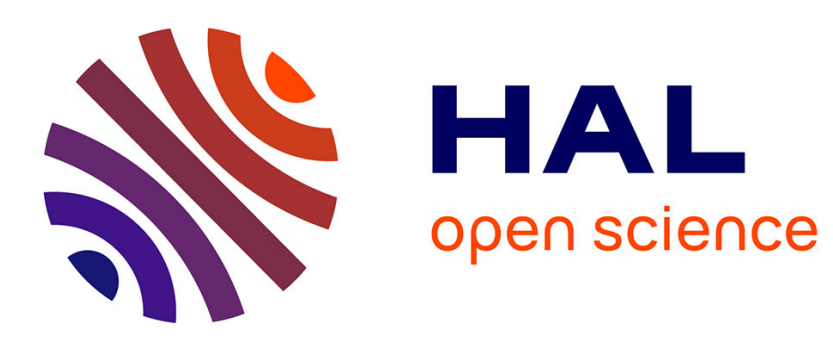

\title{
Appareillages de mesure de la piézorésistance des semiconducteurs
}

\author{
G. Weill
}

\section{To cite this version:}

G. Weill. Appareillages de mesure de la piézorésistance des semiconducteurs. Revue de Physique Appliquée, 1967, 2 (3), pp.197-202. 10.1051/rphysap:0196700203019701 . jpa-00242791

\section{HAL Id: jpa-00242791 https://hal.science/jpa-00242791}

Submitted on 1 Jan 1967

HAL is a multi-disciplinary open access archive for the deposit and dissemination of scientific research documents, whether they are published or not. The documents may come from teaching and research institutions in France or abroad, or from public or private research centers.
L'archive ouverte pluridisciplinaire HAL, est destinée au dépôt et à la diffusion de documents scientifiques de niveau recherche, publiés ou non, émanant des établissements d'enseignement et de recherche français ou étrangers, des laboratoires publics ou privés. 


\title{
BIBLIOGRAPHIE
}

[1] Gerritsen (H. J.), Helier (M. E.), " High Resolution tuned Laser Spectroscope ", Appl. Optics, Suppl. 2 on Chemical Lasers, p. 73-80, 1965.

GERRITSEN (H. J.), "Tuned-Laser Spectroscopy of Organic Vapors ", Physics of Quantum Electronics, New York, Mac Graw Hill, 1966, 581.

[2] Sakurai (K.), Shimoda (K.), " Tunable Infrared Maser Spectrometers ", Japan J. Appl. Phys., 1966, 5, 10, 938-947.

[3] BRUneT (H.), " High Resolution Spectroscopy by Zeeman-tuned Infrared Laser ", I.E.E.E. J. of Quantum Electronics, QE 2, 9, 382-384, septembre 1966.

[4] FORK (R. L.), PATEL (C. K. N.), " Broadband Magnetic Field Tuning of Optical Masers ", $A p p l$. Phys. Letters, May 1963, 2, 180-181.

[5] WhITE (H. E.), "Introduction to Atomic Spectra ", New York, Mac Graw Hill, 1964, p. 221.

[6] Moore (C. E.), "Atomic Energy Levels ", N.B.S., Washington D.C., Circular 467, août 1952.
[7] Rigrod (W. W.), "Gain Saturation and Output Power of Optical Masers ", J. Appl. Phys., septembre 1963, 34, 9, 2602-2609.

[8] SchwarTZ (L.), " Méthodes mathématiques pour les Sciences Physiques ", Paris, Hermann, 1961, p. 217-218.

[9] JAHNKE, EMDE, LöSCH, "Tables of Higher Functions ", New York, Mac Graw Hill, 1960, p. 82-94.

[10] SchwarTz (L.), loc. cit., p. 127-128.

[11] Patel (C. J. N.), "Noble Gas Optical Maser Lines at Wavelengths between $2 \mu$ and $35 \mu$ ", Phys. Rev., mars 1964, 133, $6 \mathrm{~A}, 1476-1486$.

[12] Brochard (J.), LIBERman (S.), "Émission stimulée de nouvelles transitions infrarouges de l'hélium et du néon ", C. R. Acad. Sc. Paris, juin 1965, 260, 6827-6829.

[13] LENFANT (R.), "Un polariseur dans l'infrarouge ", La recherche aéronautique, juillet-août 1961, 83, 55-57.

[14] Moret-Bailly (J.), Thèse, Paris, 10 octobre 1961, p. 73-74.

\section{APPAREILlAGES DE MESURE DE LA PIÉZORÉSISTANGE DES SEMICONDUGTEURS}

\author{
Par G. WEILL, \\ Laboratoire de Magnétisme et de Physique du Solide, G.N.R.S. (92), Meudon-Bellevue.
}

\begin{abstract}
Résumé. - L'amplification sélective de signaux alternatifs permet d'atteindre la grande sensibilité exigée par les mesures de piézorésistance. Ce résultat est obtenu soit par l'application à l'éprouvette d'une contrainte alternative, soit par l'utilisation du courant alternatif dans le circuit de mesure. Les deux appareillages décrits sont utilisables dans des domaines complémentaires. Le second, en outre, peut trouver d'utiles applications à des mesures précises de résistivité, d'effet Hall ou de magnétorésistance.
\end{abstract}

Abstract. - Measurement of piezoresistance coefficients in semiconductors requires a high sensitivity which can be achieved through selective amplification of alternating signals. Such a result has been obtained either by applying an alternating stress on the sample or by working with alternating currents in the circuits. Both techniques are described, each of which being used in the case when the other fails to give satisfactory performance. Furthermore, the lat ter may be used when precision measurements of resistivity, Hall effect or magnetoresistance are required.

I. Introduction. - Depuis les travaux de C. S. Smith [1] sur l'effet de piézorésistance dans le germanium et le silicium, l'étude de ce phénomène s'est avérée capable de fournir des indications utiles sur la structure de bandes des semiconducteurs. Rappelons qu'il s'agit de mesurer la variation des propriétés électroniques du matériau (résistivité électrique et éventuellement concentration des porteurs de charge), sous l'effet d'une contrainte mécanique.
Des mesures sous faible contrainte permettent de déterminer, d'une part les caractères de symétrie de la structure de bandes, et d'autre part des paramètres fondamentaux tels que les coefficients de pression des énergies de bord de bande et des masses effectives, et, dans le cas particulier où les bandes se déplacent linéairement avec la pression sans se déformer, les coefficients du potentiel de déformation.

G'est ainsi par exemple que Smith [1] a pu confir- 
mer la symétrie des bandes de conduction à plusieurs vallées du germanium et du silicium. De nombreux autres semiconducteurs ont été étudiés, en particulier parmi les cristaux cubiques; Potter [8] a pu préciser la structure de bande de InSb, Pikus et Bir [9] celle des chalcogénures de plomb, Groves et Paul [7] celle de l'étain gris.

Dans le cas de contraintes intenses (plusieurs milliers de bars) apparaissent éventuellement d'autres effets : désionisation des impuretés, transfert des porteurs de charge d'une bande à une autre. Nous nous limiterons ici au cas des contraintes faibles.

Du point de vue de l'appareillage de mesures, il convient de préciser quelques traits caractéristiques du phénomène étudié : lorsque la contrainte appliquée est une pression hydrostatique, le problème se ramène à la mesure d'une faible variation de résistivité, qui demeure une grandeur scalaire, dans le cas d'un cristal du système cubique. La principale difficulté expérimentale réside dans la nécessité d'éliminer l'effet de divers paramètres tels que : variations de température, gradient de température, etc., afin de mettre en évidence l'effet de la pression dont l'ordre de grandeur est souvent beaucoup plus faible. Ainsi, les valeurs observées du coefficient de pression des semiconducteurs sont de quelque $10^{-5} \mathrm{bar}^{-1}$ et atteignent exceptionnellement 1 ou $2 \times 10^{-4} \mathrm{bar}^{-1}$. Sous l'effet d'une pression de 100 bars, la variation relative de résistivité à mesurer est donc de l'ordre de quelque $10^{-3}$.

Lorsqu'il s'agit de piézorésistance axiale, les coefficients sont du même ordre de grandeur que les coefficients hydrostatiques, mais la fragilité des échantillons limite à quelques dizaines de bars la contrainte qu'il est possible de leur appliquer, si bien que la sensibilité requise est encore plus grande. Ces considérations nous ont conduit à choisir pour cette catégorie de mesures une méthode dans laquelle la contrainte appliquée est alternative.

Remarquons enfin que, pour les mesures en contrainte axiale, il importe de définir non seulement la grandeur de la contrainte, mais aussi sa direction par rapport aux axes cristallographiques de l'éprouvette, nécessairement monocristalline. Dans certaines configurations (cf. [1] et [2]), l'effort axial pourra être appliqué dans une direction perpendiculaire à celle du passage du courant.

Nous décrivons dans cet article deux catégories d'appareillages : ceux où l'éprouvette, soumise à une contrainte continue, est parcourue par un courant alternatif, et ceux où la contrainte appliquée est alternative et le courant dans l'éprouvette, continu.

II. Expériences sous contrainte axiale alternative. - II.1. Le PRINGIPE DU DISPOSITIF que nous avons adopté pour cette catégorie d'expériences a été proposé par Pollak [3] : une éprouvette monocristalline orientée, munie, comme pour une mesure de résistivité ordinaire, de deux amenées de courant et de deux prises de potentiel, est soumise à une force alternative d'amplitude $X$ connue, à la fréquence $f$. L'éprouvette est parcourue par un courant continu d'intensité $I$, dans une direction parallèle à celle de la force $X$ ( fig. 1 a). Dans ces conditions, un signal alternatif à la
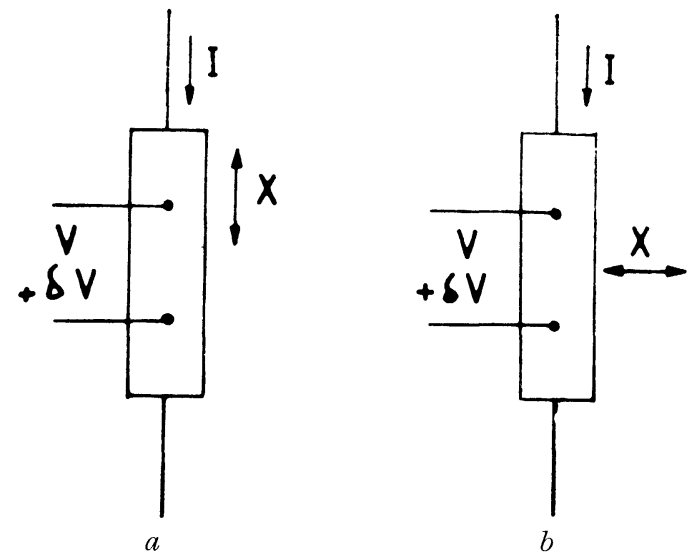

FIG. 1.

fréquence $f$ se superpose à la tension continue $V$, recueillie entre les prises de potentiel. Son amplitude $\delta V$ est proportionnelle à la variation de résistance due à l'application de la contrainte.

Notre appareillage est destiné à des matériaux de faible résistivité : tellurure de mercure et solutions solides $\mathrm{Hg}_{1-x} \mathrm{Cd}_{x} \mathrm{Te}$ avec $x<0,2$. Le signal $\delta V$ apparaît donc aux bornes d'une résistance de $10^{-3}$ à $10^{-2} \Omega$, son amplitude atteint seulement quelque $10^{-8} \mathrm{~V}$ dans les conditions les plus défavorables.

II .2. Description de l'Appareillage. - La chaîne de mesure, schématisée sur la figure 2, est constituée de la façon suivante : un transformateur à grand rapport de transformation (de 50 à 200) réalise l'adaptation d'impédance de l'échantillon à l'entrée d'un amplificateur sélectif (A.O.I.P. modèle EL 40). La sortie de cet appareil alimente, en parallèle, un voltmètre électronique et l'un des amplificateurs d'un oscilloscope à deux faisceaux.

Cette chaîne procure un gain en tension de l'ordre de $5 \times 10^{4}$, ce gain est étalonné à l'aide d'un signal d'amplitude connue à la fréquence $f$, prélevé sur un diviseur potentiométrique aux bornes d'une résistance de grandeur comparable à celle de l'échantillon.

Un blindage soigné des circuits permet de maintenir le niveau du bruit et des parasites à moins de $1 \mu \mathrm{V}$ ramené à l'entrée de l'amplificateur sélectif, soit 5 à $20 \mathrm{nV}$ à l'entrée du transformateur.

Le courant continu dans l'éprouvette est fourni par une batterie d'accumulateurs en série avec une résistance de réglage et une bobine d'inductance propre 8 henrys : l'ensemble se comporte comme un générateur d'impédance interne grande devant la résistance de l'éprouvette. 


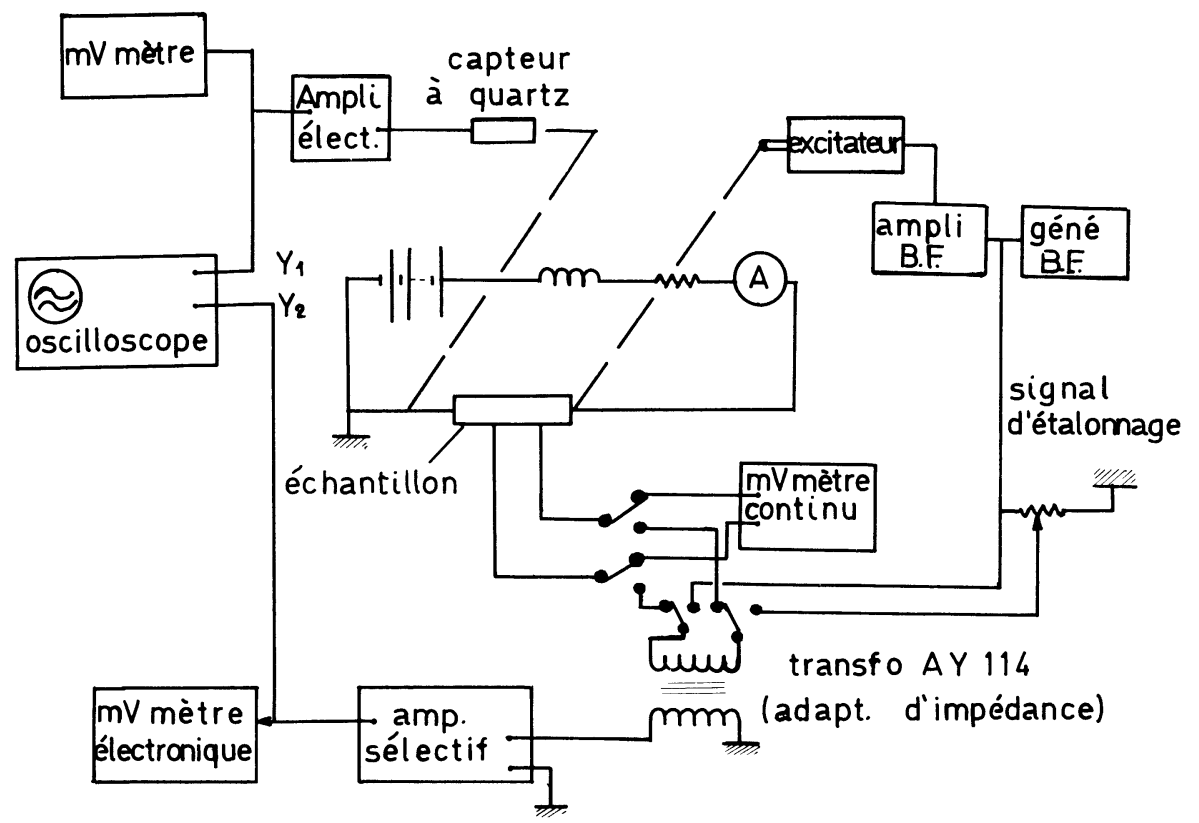

FIG. 2. - Schéma synoptique de l'appareillage de mesure en contrainte alternative.

II.3. LA DISPOSITION DES ORGANES MÉGANIQUES est représentée sur la figure 3. L'éprouvette est taillée en forme d'un parallélépipède de 8 à $12 \mathrm{~mm}$ de long et 5 à $15 \mathrm{~mm}^{2}$ de section transversale. Ses faces de bout sont soigneusement dressées par polissage.

L'une de ces faces, $F_{1}$, prend appui sur le capteur de force à quartz $Q$, par ailleurs rigidement lié au bâti $B$. La force est appliquée sur l'autre face, $F_{2}$, par l'intermédiaire d'une tige $T$ pressée sur $F_{2}$ par le ressort $\mathrm{R}$. L'effort du ressort $\mathrm{R}$ est modulé par un excitateur électromagnétique $\mathrm{E}$, agissant sur $\mathrm{T}$ par

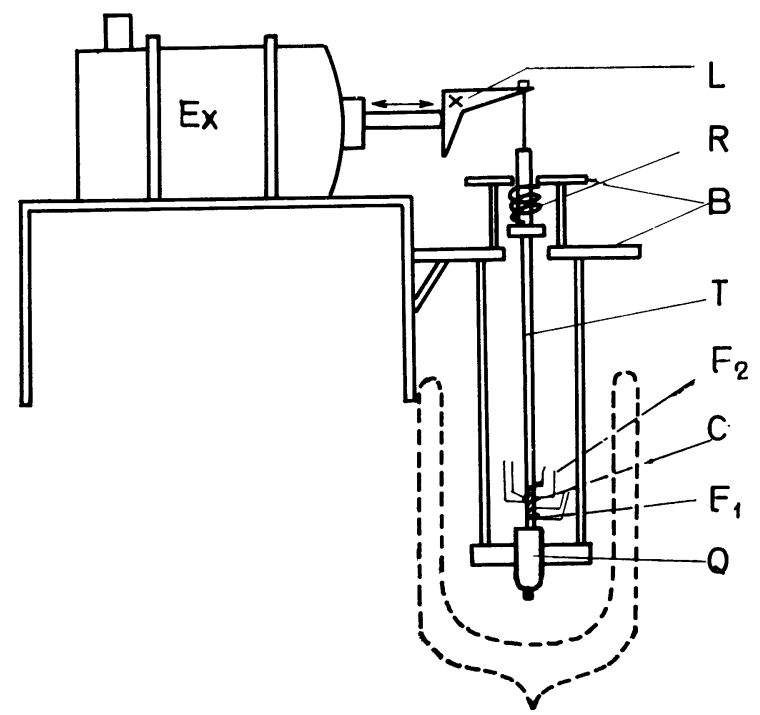

FIG. 3. - Piézorésistance en contrainte alternative. Disposition des organes mécaniques (légende dans le texte). l'intermédiaire du levier L. L'amplitude crête à crête de cette modulation, limitée en fait par la tension préalable du ressort $\mathrm{R}$, peut atteindre 20 newtons.

Le signal délivré par le capteur à quartz est mesuré par l'intermédiaire d'un amplificateur électrométrique dont la sortie alimente en parallèle un millivoltmètre et le second canal de l'oscilloscope à deux faisceaux où apparaît, sur le premier canal, la forme du signal de piézorésistance. On connaît ainsi la grandeur de la force et sa phase par rapport au signal de piézorésistance; cette phase caractérise le signe du coefficient de piézorésistance mesuré.

Pour les mesures dans une configuration telle que celle de la figure $1 b$, l'éprouvette est montée de telle manière que les faces qui s'appuient sur le capteur $Q$ et l'extrémité de la tige $\mathrm{T}$ soient des faces parallèles à la direction du courant $I$; le reste du montage est inchangé.

Enfin, la partie inférieure du dispositif peut être immergée dans un vase Dewar, permettant ainsi des mesures en fonction de la température à partir de $77 \mathrm{oK}$.

II.4. LES GARAGTÉRISTIQUES GÉNÉRALES DE L'APPAREILlAGE que nous venons de décrire peuvent se résumer comme suit.

Le fait de traiter un signal alternatif permet, grâce à l'amplification sélective, d'atteindre commodément la sensibilité requise. Cette sensibilité peut être encore augmentée en utilisant un amplificateur à détection synchrone.

La nécessité d'une stabilisation rigoureuse de la température de l'échantillon est éliminée : il est même possible d'effectuer des mesures au cours desquelles la 
température évolue lentement, le signal et la température étant enregistrés simultanément. De la même façon, la stabilisation de l'intensité dans l'éprouvette est beaucoup moins critique que dans le cas d'une mesure en courant continu. Les différences de potentiel thermoélectriques qui apparaissent si la température de l'échantillon n'est pas homogène ne constituent pas une cause d'erreurs.

Il est à remarquer que les coefficients de piézorésistance mesurés dans ces conditions sont les coefficients adiabatiques, dans la mesure où la fréquence $f$ choisie est assez grande devant l'inverse de la constante de temps thermique de l'éprouvette; cette dernière condition est parfaitement réalisée aux fréquences de 30 à $80 \mathrm{~Hz}$ que nous utilisons.

Le principal inconvénient de la méthode est qu'elle devient très difficile à appliquer en présence d'un champ magnétique, par exemple pour une mesure d'effet piézo-Hall ou de piézomagnétorésistance. En effet, l'application d'une force alternative entraîne des déplacements des circuits placés dans le champ magnétique, et les f.é.m. ainsi induites sont difficilement discernables du signal utile du fait qu'elles ont la même fréquence que lui, et des amplitudes de 10 à 100 fois plus grandes.

III. Expériences en contrainte continue. III.1. Introduction. - Pour les mesures en pression hydrostatique, la réalisation d'une contrainte alternative exigerait un appareillage relativement compliqué. Pour ces expériences, ainsi que pour toutes celles que nous effectuons en présence d'un champ magnétique, nous avons mis au point une méthode de mesure en courant alternatif dont la précision et la sensibilité sont comparables à celles des méthodes classiques utilisées en courant continu ou haché [4].

III.2. LE PRINGIPE DE LA MÉTHODE est illustré par la figure 4. L'éprouvette de mesure est placée en série avec une résistance de référence $R_{0}$. Les prises de potentiel de l'éprouvette et les extrémités de $R_{0}$ sont reliées aux primaires des transformateurs $T_{1}$ et $T_{2}$ respectivement. La différence de potentiel qui apparaît aux bornes du secondaire de $\mathrm{T}_{1}$ est opposée à une fraction $x(0<x<1)$ de celle qui apparaît aux bornes de $T_{2} . x$ est ajusté de manière que le courant soit nul dans le secondaire de $T_{1}$. Le courant de déséquilibre du pont ainsi constitué est amplifié sélectivement, et sa phase est comparée, sur un oscilloscope par exemple, à celle du courant $i$ dans le circuit principal. Lorsque l'équilibre est réalisé, on a, si $n_{1}$ et $n_{2}$ sont les rapports de transformation de $T_{1}$ et $T_{2}$ :

$$
\frac{V}{i} n_{1}=x R_{0}^{\prime} n_{2}
$$

où $R_{0}^{\prime}$ est une résistance qui dépend de $R_{0}$, de la résistance du primaire de $\mathrm{T}_{2}$ et de l'impédance, vue des bornes $\mathrm{A}$ et $\mathrm{B}$ du circuit secondaire de $\mathrm{T}_{2}$. En première approximation, le facteur $R_{0}^{\prime} n_{2} / n_{1}$ ne dépend que des caractéristiques d'éléments passifs : c'est la constante d'étalonnage de l'appareil, qui peut être déterminée en substituant à l'éprouvette une résistance de valeur connue.

III.3. RÉAlisation PRATiQUe. - Dans l'appareillage réalisé, le générateur $\mathrm{G}$ est constitué d'un générateur B.F. pilotant un amplificateur capable de délivrer environ $10 \mathrm{~W}$ dans une impédance de quelques ohms. (Nous avons appliqué la méthode à la mesure de résistances comprises entre $10^{-4}$ et $1 \Omega$.) La fréquence de travail a été fixée à $80 \mathrm{~Hz}$ en fonction de critères tels que : bande passante des transformateurs, sélectivité optimale de l'amplificateur de zéro, influence des réactances parasites. Les transformateurs $T_{1}$ et $T_{2}$ sont des pièces détachées disponibles dans le commerce (Lie-Belin AY 114) pour lesquels $n$ peut être choisi entre 30 et 200 grâce à un primaire à enroulements multiples.

Le détecteur de zéro est un amplificateur A.O.I.P. modèle EL 40. Le signal de référence de phase est pris aux bornes du générateur B.F. pilotant $\mathrm{G}$.

Le diviseur $\rho$ est constitué de deux potentiomètres hélicoïdaux à 10 tours de 10000 et $1000 \Omega$ respectivement. Ils sont munis de boutons de commande

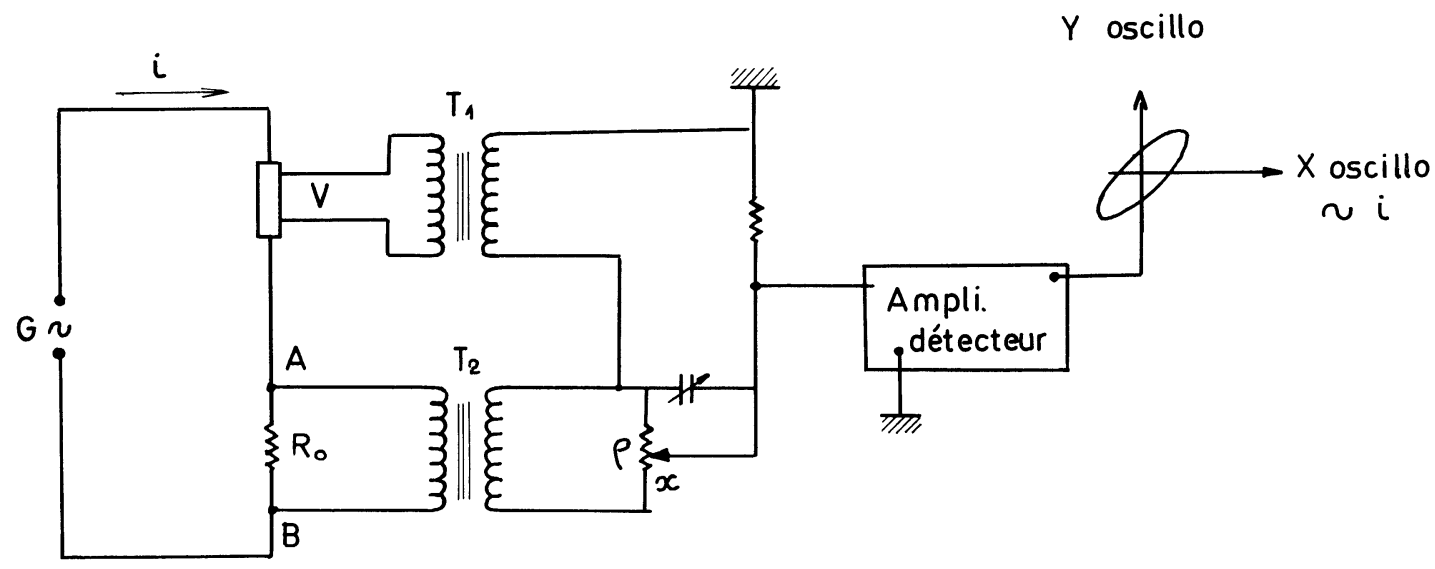

FIG. 4. - Mesure de résistivité en courant alternatif. Schéma de principe. 
gradués où s'affiche la valeur $x$. Un commutateur à galettes permet de choisir 6 combinaisons des valeurs de $R_{0}$ et de $n_{2}$ de manière à adapter l'étendue de mesure de l'appareil à la résistance de l'échantillon en expérience. Enfin, à l'aide d'un inverseur, il est possible de retourner le branchement des prises de potentiel aux bornes de $T_{1}$ : cela est nécessaire par exemple lors d'une mesure d'effet Hall où la tension à mesurer est en phase ou en opposition avec $i$ selon le sens du champ magnétique appliqué.

iII.4. Performances et limitations. - Comme pour toute méthode de zéro, la précision de la mesure est affectée uniquement par la stabilité et la définition des éléments passifs que sont les résistances et les transformateurs, et par la sensibilité du détecteur de zéro. A cet égard, il convient de remarquer que les impédances des différents éléments du circuit ne sont pas purement actives, si bien que le courant de déséquilibre a une composante en quadrature avec $i$. Le zéro est défini avec une plage d'incertitude d'autant plus large que cette composante est plus importante. On améliore la sensibilité en compensant la composante en quadrature à l'aide d'un condensateur variable disposé en parallèle avec la résistance $\rho$.

Le détecteur utilisé a un bruit de fond de l'ordre de $1 \mu \mathrm{V}$, ce qui autorise, étant donné la valeur de $n$, une sensibilité de $10^{-4}$ si le courant dans l'échantillon est tel que la tension à ses bornes soit de $0,1 \mathrm{~V}$.

De même que dans le montage décrit dans la section II, la sensibilité peut être améliorée d'un facteur 10 environ par l'emploi comme détecteur d'un amplificateur « lock in ».

Lorsque l'échantillon, et donc une partie du circuit, est placé dans un champ magnétique intense (mesures d'effet Hall ou de magnétorésistance), les forces de Laplace provoquent des vibrations mécaniques à la fréquence du courant $i$. Il en résulte l'apparition de f.é.m. induites à la même fréquence, qui peuvent compromettre l'utilisation de la méthode si l'on ne prend pas soin de fixer rigidement l'échantillon et toutes les connexions parcourues par un courant d'intensité notable.

III.5. Appligations A la PiÉzorésistance. - Pour appliquer la méthode décrite ci-dessus à des mesures, sous pression hydrostatique, de la résistivité et de l'effet Hall [5], [6], l'échantillon est enfermé dans une enceinte de métal amagnétique (ARGAP) pressurisée à l'aide d'une bouteille de gaz comprimé du commerce (azote ou hélium selon la température). L'ensemble est représenté sur la figure 5 . Les passages électriques isolés, étanches à la pression, sont constitués, pour les expériences à température ordinaire, d'un bouchon d'araldite traversé par les fils conducteurs. Pour les basses températures (azote liquide), les passages sont réalisés à l'aide de fils "Thermocoax » soudés à

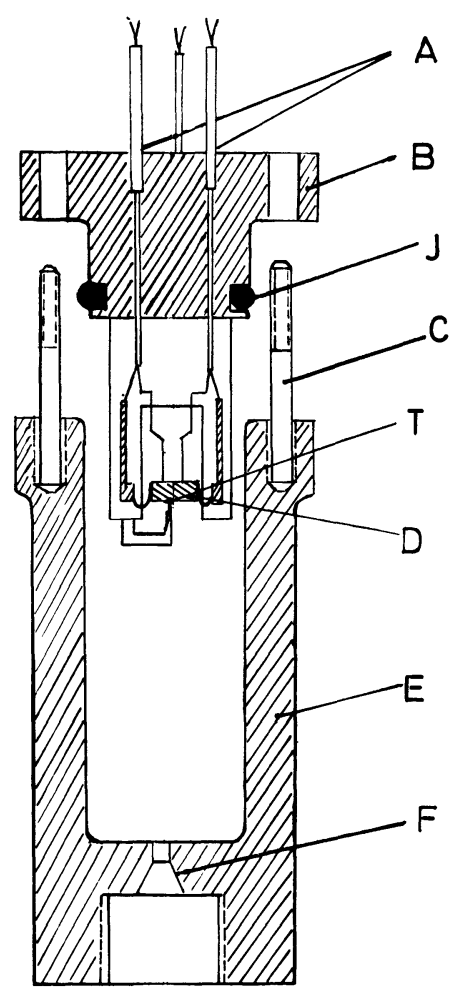

FIG. 5. - Enceinte pour mesure sous pression jusqu'à 200 bars. - A : passage Thermocoax. - B : couvercle. $-\mathrm{C}$ : goujons de fixation. - D : éprouvette. - $\mathrm{E}$ : corps de bombe. $-\mathrm{F}$ : arrivée du gaz sous pression. - $\mathrm{T}$ : thermocouple. $-\mathrm{J}$ : joint torique.

l'étain dans des canaux ménagés à travers la paroi de l'enceinte $\left.{ }^{\mathbf{1}}\right)$.

Pour les mesures sous contrainte axiale, le montage utilisé est analogue à celui de la figure 3 . La force est appliquée par des poids posés sur un plateau monté à l'extrémité de la tige $\mathrm{T}$.

Pour l'ensemble des mesures sous contrainte continue, le choix du courant alternatif a permis d'atteindre une bonne sensibilité et d'éliminer les erreurs dues aux effets thermoélectriques et thermomagnétiques. Mais il ne lève pas la sujétion d'une stabilisation rigoureuse de la température. Pratiquement, il est nécessaire, surtout dans le cas de " petits » effets, que l'éprouvette soit en équilibre thermique avec un bain constituant un point fixe $\left(\mathrm{N}_{2}\right.$ liquide bouillant, glace fondante, etc.). Remarquons enfin que la mesure sous contrainte continue conduit aux valeurs isothermes des coefficients de piézorésistance contrairement à la précédente qui donne les valeurs adiabatiques.

IV. Conclusions générales. - Nous avons décrit deux catégories d'appareillages de mesure qui se

(1) Nous remercions M. Lallemand, du Laboratoire des Hautes Pressions du C.N.R.S., pour les conseils qu'il nous a donnés à ce sujet. 
complètent mutuellement dans le cas de la piézorésistance.

On sait en effet [2] que pour déterminer les 3 coefficients indépendants du tenseur de piézorésistance d'un cristal cubique, une au moins des trois mesures nécessaires doit être effectuée dans la configuration $b$ ( fig. 1) ou la configuration hydrostatique. Cette dernière solution est préférable car les conditions expérimentales dans la configuration $b$ ne permettent d'atteindre qu'une précision médiocre.

Nous utilisons actuellement les méthodes décrites dans cet article à l'étude de la piézorésistance du tellurure de mercure et des alliages $\mathrm{Hg}_{1-x} \mathrm{Cd}_{x} \mathrm{Te}$. Nous mentionnerons brièvement les principaux résultats obtenus :

- Pour le tellurure de mercure de type $p$, la piézorésistance axiale est anisotrope, ce qui peut être attribué à la présence de maxima de la bande de valence situés en des points différents de $\mathbf{k}=0$;

- Pour le tellurure de mercure de type $n$, on observe un effet de piézorésistance axiale, indépendant de la direction de la contrainte. Cela signifie que, malgré l'anisotropie élastique de $\mathrm{HgTe}$, le potentiel de déformation peut être décrit par un et non deux coefficients. Ce résultat est à rapprocher de celui qu'a établi D. G. Thomas [10] pour le tellurure de cadmium, et qu'il a attribué dans ce cas à la levée de dégénérescence de la bande de valence;

- Enfin, pour $\mathrm{HgTe}$ et les solutions solides $\mathrm{Hg}_{1-x} \mathrm{Cd}_{x} \mathrm{Te}$, nous avons pu relier les effets observés en pression hydrostatique au coefficient de pression de l'intervalle $\Gamma_{7}-\Gamma_{8}^{-}$, que nous avons évalué à

$$
(12 \pm 2) \times 10^{-6} \mathrm{eV} / \text { bar }[6]
$$

Remarquons enfin que la méthode décrite dans la section III est d'une utilité plus générale. Elle s'avère avantageuse lorsqu'il s'agit de mesurer de petites variations d'une impédance sous l'influence d'une grandeur extérieure quelle qu'elle soit. C'est le cas par exemple des mesures de l'effet Hall ou de la magnétorésistance de matériaux où la mobilité des porteurs de charge est petite.

Manuscrit reçu le 21 avril 1967.

\section{BIBLIOGRAPHIE}

[1] Sмiтh (C. S.), Phys. Rev., 1954, 94, 42.

[2] Keves (R. W.), Solid State Physics, vol. 11, p. 149, Academic Press, New York, 1960.

[3] Poliak (M.), Rev. Sci. Inst., 1958, 29, 639.

[4] DauphineE (T. M.), MoOser (E.), Rev. Sci. Inst., $1955,26,660$.

[5] Lali.emand (M.), Weili (G.), LAcam (A.), RoDOT (M.), C. R. Acad. Sci., 1965, 260, 4469.
[6] Weiri, (G.), Verié (C.), C. R. Acad. Sci., 1966, 263, 463.

[7] Groves (S.), Paul (W.), C. R. Conf. Int. Phys. Semicond., p. 41, Dunod, Paris, 1964.

[8] Potter (R. F.), Phys. Rev., 1957, 108, 652.

[9] BIR (G. L.), Pikus (G. E.), Sov. Phys. Solid State, 1963, 4, 1640.

[10] Thomas (D. G.), J. Appl. Physics, 1961, 32, 2298. 